\title{
Color Image Enhancement using Subsampled Channel Difference
}

\author{
Seungjong Kim \\ Department of Computer Science \& Information Systems, \\ Hanyang Women's University, Seoul, Korea \\ jkim@hywoman.ac.kr
}

\begin{abstract}
To minimize the cost of color restoration, current digital cameras use color filter array (CFA) instead of beam splitters of full-color camera. In this paper, a new demosaicking method is proposed which has three stages: (1) green channel restoration, (2) red/blue channel restoration, and (3) three channels refinement. As green channel has more information than that of red/blue, green channel restoration is performed before red/blue channel restoration. The proposed method uses channel difference information and parameter applied weighted average approaches. Experimental results indicate that the proposed method provides reliable results in terms of objective and subjective metrics.
\end{abstract}

Keywords: Bayer CFA, color interpolation, channel difference, image quality assessment

\section{Introduction}

The full color image is generally composed of multi-color channels, and at least three color sensors are required to show color image [1]. In electronic color imaging, it is necessary to simultaneously acquire image data in three color planes (Red, Green, and Blue). Although the digital cameras resolution has been increasing, the size of CMOS sensors could not be increased accordingly [2]. However, the CMOS sensors are still requested to acquire huge number of pixels [3,4]. Therefore, the quantity of light obtained in the same time by each unit is remarkably reduced. This degradation of light sensitivity by each unit can cause downgrade in restored image quality, particularly in dark area [5-6]. Therefore, the images obtained by above cameras can have noisy and blurring effect, and may require a long exposure time to obtain enough light to achieve acceptable images.

When a color image is acquired using a CFA, it is needed to populate the $\mathrm{R}, \mathrm{G}$, and $\mathrm{B}$ intensities so that there is a reconstruct of all 3 color intensities for each sensor position. Once the population is accomplished, each pixel has 3 color intensities and can be manipulated by various image processing methods. To reduce the cost, currently available digital cameras uses color filter array (CFA) to obtain a color image. Therefore, each pixel only captures one color, and this process is mosaicking, and the opposite process is demosaicking (or color interpolation) [7-9]. The opposite process stands for reconstructing missing two colors information. After capture, the signal of each pixel is read out sequentially; digitized and stored in memory. The original data for each pixel only contains information about one color, depending on which filter is positioned over that pixel. However, information for all three primary colors is needed to construct a color image. The missing information is extracted based on the information gathered by the neighboring pixels. This is called "color interpolation". There are a variety of interpolation methods, such as " Nearest Neighbor Interpolation", "Bilinear Interpolation", and "Bi-cubic Interpolation", etc. These simple methods are described in the literature. The quality of the final image depends in large part on which algorithm is used. For this reason many camera manufacturers consider their best color interpolation 
algorithms to be trade secrets and do not publish them. The colors values obtained through the color interpolation process are called native colors. Due to the spectral characteristics of the optics (lens, filters) used in the system, the lighting conditions (such as daylight, fluorescent, and etc.) of the scene, and the characteristics of the color filters of the sensor, the native RGB data may not provide a faithful color rendition. One of mostly successfully CFAs is Bayer pattern CFA [10]. In Bayer pattern CFA, each pair has four pixels, two are from green channel and the other two are from red/blue channel, respectively. Figure 1 shows an example of $5 \times 5$ Bayer CFA pattern where red is located in center $(i, j)$ position.

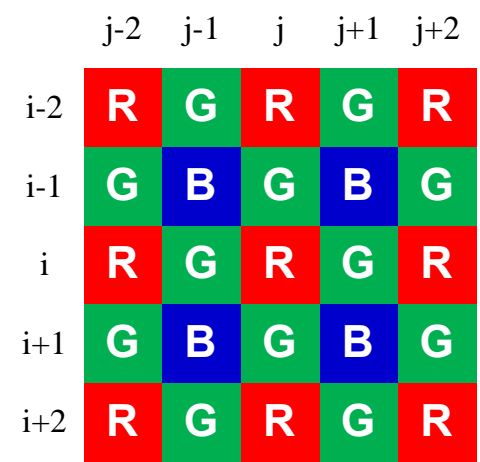

\section{Figure 1. 5×5 Bayer CFA Pattern Example where Red Channel is Located in Center (I, J) Position}

There have been several demosaicking methods with diverse degree of complexity and accuracy. One of simplest methods is bilinear (or bicubic) interpolations which are considered to be the straightforward approach where the missing color information is reconstructed in a single color component independently [11-14]. However, the high frequency component cannot be well restored. One of the most well-known direction based method is adaptive color plane interpolation algorithm $[15,16]$. By considering inter-channel correlation, some advanced methods were presented to try to preserve edge details and provide better color demosaicking result [17-20]. For instance, the ECI method is proposed to achieve full color image by restoring the color channels between green and red/blue components. Based on effective color interpolation approach, other demosaicking methods were proposed. The primary-consistent soft-decision approach removes color components artifacts by undertaking the same demosaicking direction for each color component of a pixel. These all methods were based on adaptive color plane interpolation algorithm with more accurate edge direction computing.

In this paper, a new color interpolation method is proposed which has three stages. In the first stage, the green channel is restored using color channel difference. As the green channel has twice information than that of red or blue, the restored green channel information is used for red or blue channel interpolation. After fully populating red, green and blue information, refinement process is applied to remove outlier pixels.

This paper is arranged as follows. Section 2 introduces color restoration technique based on subsampled channel difference. Section 3 describes simulation results in terms of objective metrics and subjective quality comparison. Finally, conclusion remarks are provided in Section 4. 


\section{Proposed Method}

The proposed method restored the green channel first. Then other color components (red and blue) are restored using already restored green channel. As the green channel has more usable information, employing restored green channel for red/blue restoration is helpful. For example, it is assumed that the center pixel is located at $(i, j)$ and the center pixel color component is red. There are four edge directions, $0^{\circ}$ (horizontal: $H$ ), $90^{\circ}$ (vertical: $V$ ) $45^{\circ}$ (upper-right diagonal: $U R$ ), and $135^{\circ}$ (upper-left diagonal: $U L$ ) gradients at $(i, j)$ locations. Then, $H_{i, j}, V_{i, j}, U R_{i, j}$, and $U L_{i, j}$, are computed as follows.

$$
\begin{aligned}
& H_{i, j}=\left|G_{i, j-1}-G_{i, j+1}\right|+\alpha\left|R_{i, j-2}-2 R_{i, j}+R_{i, j+2}\right| \\
& V_{i, j}=\left|G_{i-1, j}-G_{i+1, j}\right|+\alpha\left|R_{i-2, j}-2 R_{i, j}+R_{i+2, j}\right| \\
& U R_{i, j}=\frac{\left|G_{i, j-1}-G_{i-1, j}\right|+\left|G_{i, j+1}-G_{i+1, j}\right|}{2}+\alpha\left|R_{i-2, j+2}-2 R_{i, j}+R_{i+2, j-2}\right| \\
& U L_{i, j}=\frac{\left|G_{i, j-1}-G_{i+1, j}\right|+\left|G_{i, j+1}-G_{i-1, j}\right|}{2}+\alpha\left|R_{i-2, j-2}-2 R_{i, j}+R_{i+2, j+2}\right|
\end{aligned}
$$

In Equation (1), $B_{i, j}$ and $G_{i, j}$ are still unknown, and only $R_{i, j}$ are available. Parameter $\alpha$ is weight value. Figure 2 shows four cases of edge directions.

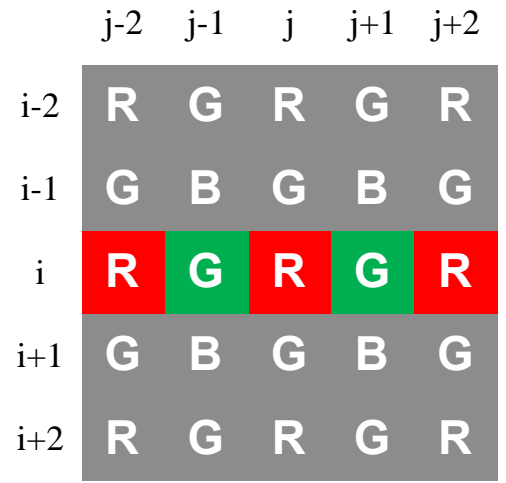

(a)

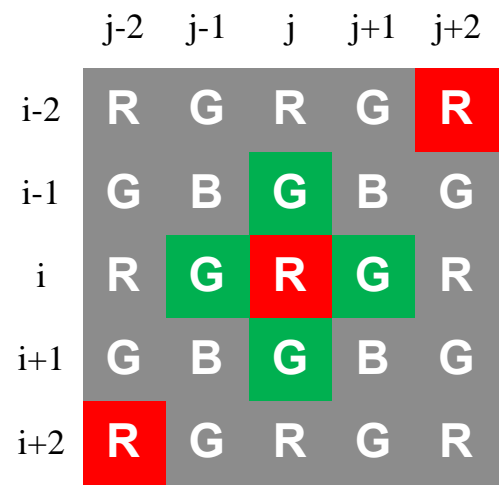

(c)

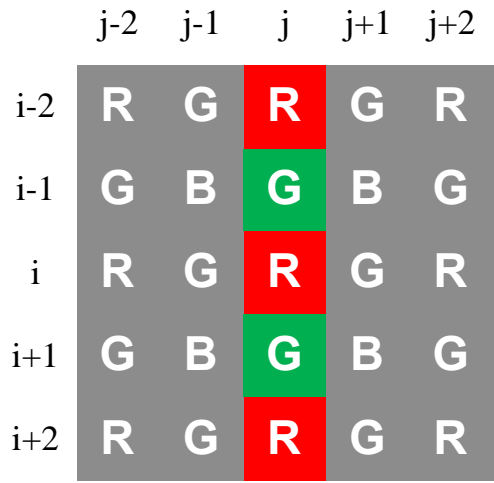

(b)

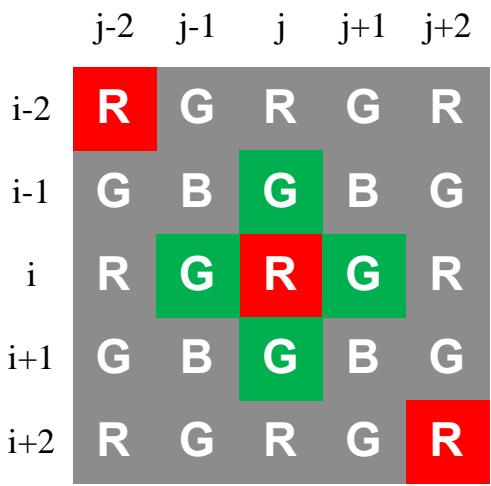

(d)

Figure 2. Four Cases of Edge Directions for Restoration: (a) Horizontal $(H)$, (b) Vertical ( $V$, (c) Upper-Right (UR), and (D) Upper Left (UL)

If $\alpha$ is smaller (or bigger) than 1 , then green pixels have more (or less) influence to determine direction. It is assumed that the missing green $(M G)$ components are obtained by following equations: 


$$
\begin{aligned}
& M G_{i, j}^{H}=\frac{G_{i, j-1}+G_{i, j+1}}{2}-\beta\left(\frac{R_{i, j-2}-2 R_{i, j}+R_{i, j+2}}{4}\right) \\
& M G_{i, j}^{V}=\frac{G_{i-1, j}+G_{i+1, j}}{2}-\beta\left(\frac{R_{i-2, j}-2 R_{i, j}+R_{i+2, j}}{4}\right) \\
& M G_{i, j}^{U R}=\frac{G_{i-1, j}+G_{i+1, j}+G_{i, j-1}+G_{i, j+1}}{4}-\beta\left(\frac{R_{i-2, j+2}-2 R_{i, j}+R_{i+2, j-2}}{4}\right) \\
& M G_{i, j}^{U L}=\frac{G_{i-1, j}+G_{i+1, j}+G_{i, j-1}+G_{i, j+1}}{4}-\beta\left(\frac{R_{i-2, j-2}-2 R_{i, j}+R_{i+2, j+2}}{4}\right)
\end{aligned}
$$

Similarity, $\beta$ is a weight parameter. As described above, there are four directions, $H, V$, $U R$, and $U L$. Parameter $P$ is obtained as,

$$
P_{i, j}=\min \left(H_{i, j}, V_{i, j}, U R_{i, j}, U L_{i, j}\right)
$$

When $P_{i, j}=H_{i, j}$, it is assumed that the green pixel is interpolated horizontally and $M G_{i, j}^{H}$ is considered for restoration. In addition, when $P_{i, j}=U R_{i, j}$, the green pixel is interpolated upper right direction and $M G_{i, j}^{U R}$ is considered for restoration.

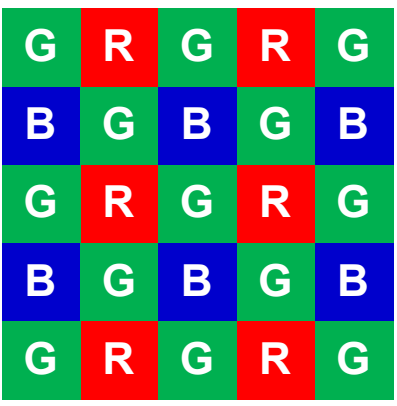

(a)

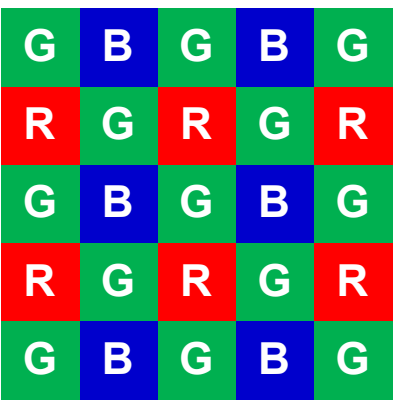

(b)

\section{Figure 3. Two $5 \times 5$ Blocks of Bayer CFA: (a) Red Pixels are Horizontally Located, (b) Blue Pixels are Horizontally Located}

After the green channel is restored, remained red and blue channels are restored. As the interpolated green channel is reliable, the green channel information is adopted for investigating red and blue channels. The unknown red/blue channels are components at green CFA sampling positions are populated. Figure 3 shows two examples that a green pixel is positioned at the center of $5 \times 5$ block. The only difference is that the red pixels are horizontally located in Figure 3(a) while the blue pixels are horizontally located in Figure 3(b). For the blue channel interpolation at red location (Figure 1 case), information located in the diagonal direction could be good estimates for interpolation. The red and blue information are populated by Equations (4) and (5), respectively. In this step, $\lambda_{l}$ and $\lambda_{2}$ are weight parameters for left and right, or upper and lower directions.

$$
\begin{gathered}
R_{i, j}=G_{i, j}+\lambda_{1}\left(R_{i, j-1}-G_{i, j-1}\right)+\lambda_{2}\left(R_{i, j+1}-G_{i, j+1}\right) \\
B_{i, j}=G_{i, j}+\lambda_{1}\left(B_{i-1, j}-G_{i-1, j}\right)+\lambda_{2}\left(B_{i+1, j}-G_{i+1, j}\right)
\end{gathered}
$$

When red/blue components are positioned in green channel, there are two scenarios. To obtain red and blue channels in Figure 3(a), the bicubic interpolation method is recommended. The red channel is horizontally interpolated while blue channel is vertically interpolated:

$$
R_{i, j}=G_{i, j}+\frac{-\left(R_{i-3, j}-G_{i-3, j}\right)+3\left(R_{i-1, j}-G_{i-1, j}\right)+3\left(R_{i+1, j}-G_{i+1, j}\right)-\left(R_{i+3, j}-G_{i+3, j}\right)}{4}
$$




$$
B_{i, j}=G_{i, j}+\frac{-\left(B_{i, j-3}-G_{i, j-3}\right)+3\left(B_{i, j-1}-G_{i, j-1}\right)+3\left(B_{i, j+1}-G_{i, j+1}\right)-\left(B_{i, j+3}-G_{i, j+3}\right)}{4}
$$

For the case of Figure 3(b), the red channel is interpolated vertically and the blue channel is interpolated horizontally.

$$
\begin{aligned}
& R_{i, j}=G_{i, j}+\frac{-\left(R_{i, j-3}-G_{i, j-3}\right)+3\left(R_{i, j-1}-G_{i, j-1}\right)+3\left(R_{i, j+1}-G_{i, j+1}\right)-\left(R_{i, j+3}-G_{i, j+3}\right)}{4} \\
& B_{i, j}=G_{i, j}+\frac{-\left(B_{i-3, j}-G_{i-3, j}\right)+3\left(B_{i-1, j}-G_{i-1, j}\right)+3\left(B_{i+1, j}-G_{i+1, j}\right)-\left(B_{i+3, j}-G_{i+3, j}\right)}{4}
\end{aligned}
$$

While all channels (Red, Green, and Blue) can be restored by the proposed method, color artifacts are yet to be addressed. Therefore, refinement process is usually executed to further enhance the performance of the interpolation in various demosaicking methods. In this paper, the refinement process of Chen and Chang [8] is adopted for refining the green pixel values of an image, and the detailed description is as follows.

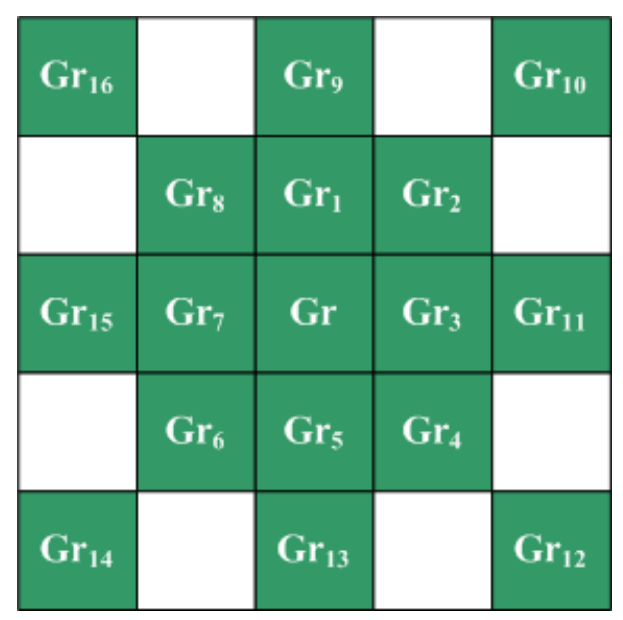

Figure 4. Color Difference Plane of Gr

After all the pixel values of an image are restored by interpolation, each location has an $R, G$ and $B$ pixel value. The refinement process begins by calculating the color difference $\mathrm{Gr}(\mathrm{G}-\mathrm{R})$ values of each point. The color difference plane of $\mathrm{Gr}$ is shown in Figure 4 and the Gr values are estimated with adjacent points using the Equation (10). $C b_{k}$ represent color difference correlations with adjacent points and are defined as Equation (11).

$$
\begin{gathered}
G r=\sum_{j=1}^{8} W_{j} G r_{j}, \quad W_{j}=C b_{j} / \sum_{k=1}^{8} C b_{k} \\
C b_{1}=1 /\left[1+\left(\left|G r_{1}-G r_{5}\right| / 2\right)+\left(\left|G r-G r_{9}\right| / 2\right)\right] \\
C b_{2}=1 /\left[1+\left(\left|G r_{2}-G r_{6}\right| / 2 \sqrt{2}\right)+\left(\left|G r-G r_{10}\right| / \sqrt{2}\right)\right] \\
C b_{3}=1 /\left[1+\left(\left|G r_{3}-G r_{7}\right| / 2\right)+\left(\left|G r-G r_{11}\right| / 2\right)\right] \\
C b_{4}=1 /\left[1+\left(\left|G r_{4}-G r_{8}\right| / 2 \sqrt{2}\right)+\left(\left|G r-G r_{12}\right| / \sqrt{2}\right)\right] \\
C b_{5}=1 /\left[1+\left(\left|G r_{1}-G r_{5}\right| / 2\right)+\left(\left|G r-G r_{13}\right| / 2\right)\right] \\
C b_{6}=1 /\left[1+\left(\left|G r_{2}-G r_{6}\right| / 2 \sqrt{2}\right)+\left(\left|G r-G r_{14}\right| / \sqrt{2}\right)\right] \\
C b_{7}=1 /\left[1+\left(\left|G r_{3}-G r_{7}\right| / 2\right)+\left(\left|G r-G r_{15}\right| / 2\right)\right] \\
C b_{8}=1 /\left[1+\left(\left|G r_{4}-G r_{8}\right| / 2 \sqrt{2}\right)+\left(\left|G r-G r_{16}\right| / \sqrt{2}\right)\right]
\end{gathered}
$$

A new Gr is obtained by applying Equation (10) with the weighted values of the adjacent eight points. A new Gb can also be obtained using the same method. The new $\mathrm{Gr}$ 
and $\mathrm{Gb}$ values are then computed backwards, respectively, to find the two new green pixel values, which are added and averaged to produce the adjusted green pixel value.

\section{Experimental Results}

The proposed approach is tested on 20 LC images (\#51-70). Figure 5 shows 20 selected LC images. All test images are initially executed by Bayer CFA pattern and then interpolated back to three color components using color interpolation methods. The reconstructed images are compared to the original image with several objective metrics such as color peak signal to noise ratio (CPSNR), color mean squared error (CMSE), and S-CIELAB. It is noted that pixels within eleven pixel distance from the border area are not examined in the experiments. The first two metrics are CPSNR and CMSE. The CMSE is calculated as,

$$
C M S E=\frac{1}{3 H W} \sum_{i=\{R, G, B\}} \sum_{y=1}^{H} \sum_{x=1}^{W}[\operatorname{ori}(x, y, i)-\operatorname{rec}(x, y, i)]^{2} .
$$

In Equation (12), ori and rec stand for the original image and the demosaicked images. Parameters $x, y$, and $i$ are horizontal position, vertical position, and color channel number, respectively. $H$ is the image height and $W$ is the image width of the tested image. For LC dataset, $H$ is 540 and $W$ is 720 . Once CMSE is computed, then CPSNR can be computed as,

$$
C P S N R=20 \log _{10}\left(\frac{\text { peak }}{\sqrt{\text { CMSE }}}\right) .
$$

In Equation (13), peak is 255. The predetermined parameters $\alpha=1.2, \beta=0.8, \lambda_{1}=0.9$ and $\lambda_{2}=0.9$ are used in this paper. Figure 6 shows the process of generating CFA image. A pair of Bayer CFA pattern has four pixels and CFA pattern may be three candidates: RGGB, RRGB, and RGBB. For example, if RGGB is obtained, it has two green pixels, one red pixel and one blue pixel. Therefore, the red channel is obtained by applying cyan filter, and the blue channel is obtained by applying green filter. After that, the rest of signals are green channel [19].

Result images are shown in Figures 7-11, where five images are displays: original image, bilinear interpolation image, proposed method, difference between bilinear and the original one, and the difference between the proposed method image and the original one.

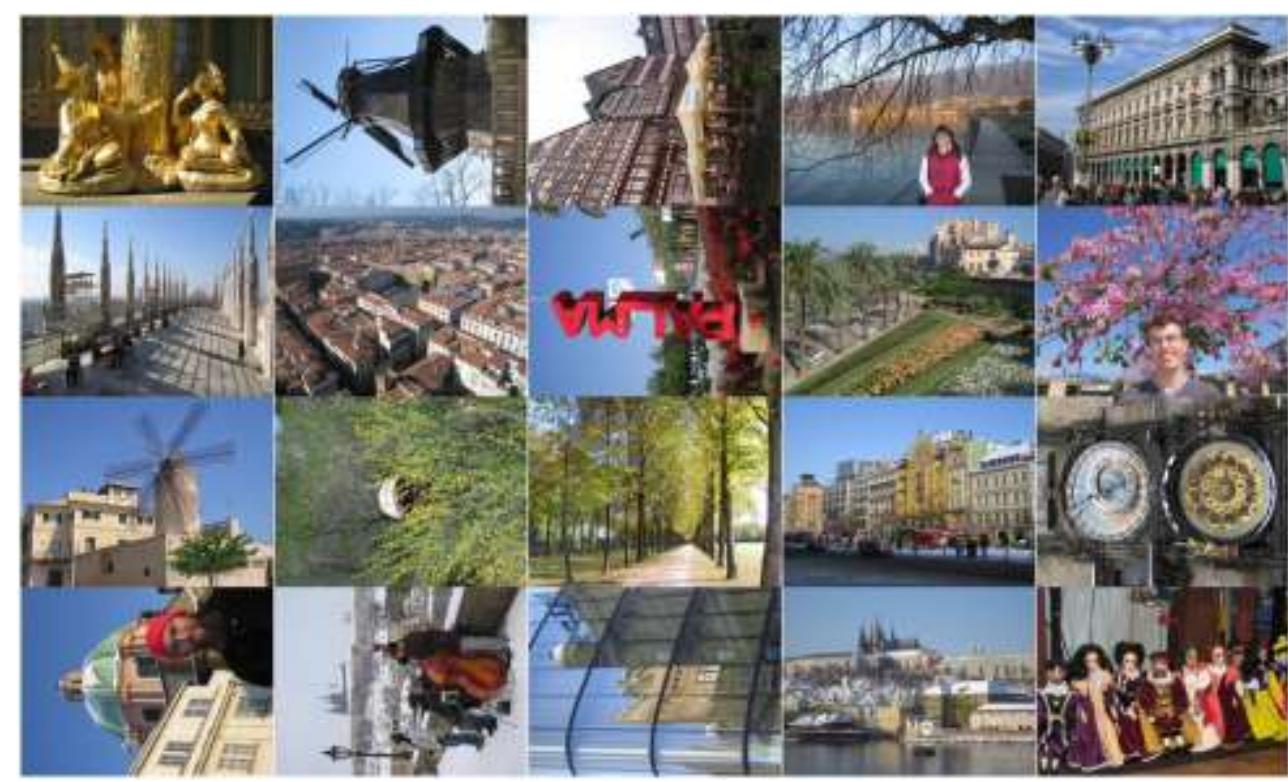

Figure 5. 20 Test Images, LC Dataset \#51-70 


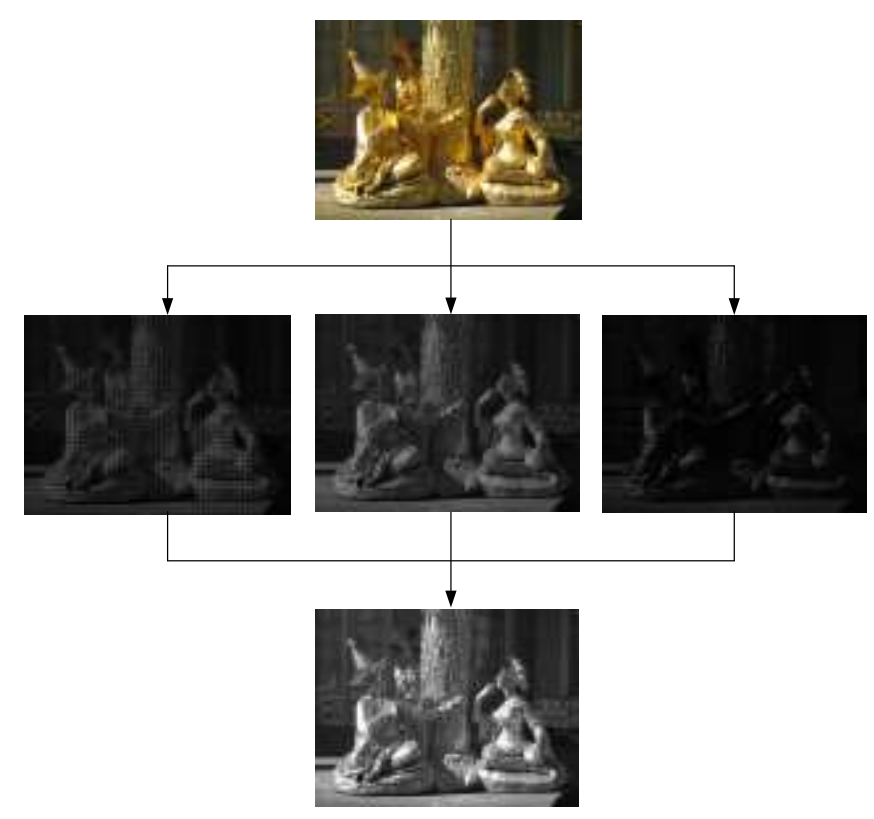

Figure 6. Process of Generating CFA Image

Figure 7 shows simulation results comparison on LC \#51 image. As can be seen in the figures, the proposed approach well restores image similar to the original one. This can be found from Figures 7(c) and 7(e). On the other hand, the benchmark method shows undesirable color errors as shown in Figure 7(b) and Figure 7(d). The same simulation was tested on LC \#52-55 images. All images indicate that the proposed method outperforms the benchmark in terms of subjective performance comparison.

Figure 8 shows simulation results comparison using LC \#52 image. The proposed method well reconstructed image similar to the original one. However, bilinear interpolation result shows unwanted color artifacts, as shown in Figure 8(b). Figure 9 shows simulation result with LC \#53 image. The original image has mosaic pattern, and high frequency components are in the image. Figure 9(b) shows false color and blurred. On the other hand, the proposed method shows favor result as shown in Figure 9(c). Figure 10 shows results from LC \#54 image. Figure 10(d) shows difference image between original and bilinear interpolation. It is obvious that bilinear interpolation results blur and false color artifacts. However, the proposed method did not reveal color errors.

Figure 11 shows results from LC \#55 image. The bilinear interpolation suppressed high frequency component, therefore result image looks like low pass filtered image. Figure 11(d) shows difference image between original and bilinear interpolation. Figure 11(e) represents difference image between original and proposed method, and shows the least differences, which indicates the proposed method is quite identical to the original one. As can be seen in all simulation figures, the proposed approach achieved similar result with the original image, while bilinear interpolation result shows blurred image. In addition, various false colors are shown throughout the restored image. Moreover, by proposing a new edge direction determination rule, good restoration performance is achieved. 


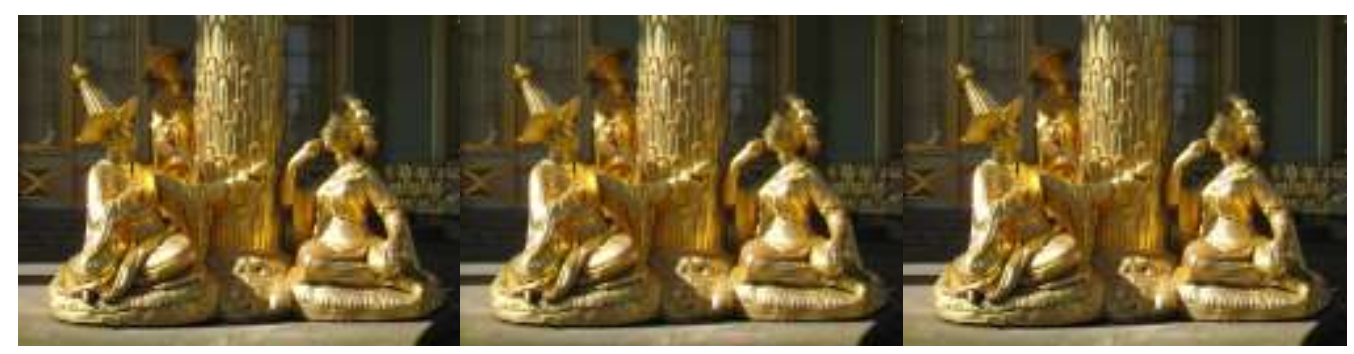

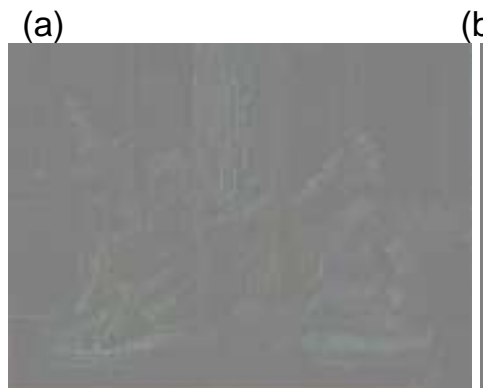

(d) (b)

(c)

Figure 7. Simulation Results: (a) Original LC \#51 Image, (b) Bilinear Interpolation, (c) Proposed Method, (d) Difference Image between (a) and (b), and (e) Difference Image between (a) and (c)

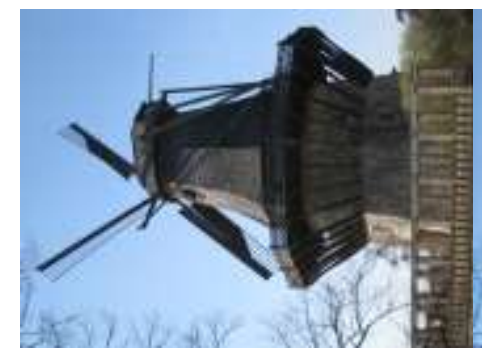

(a)

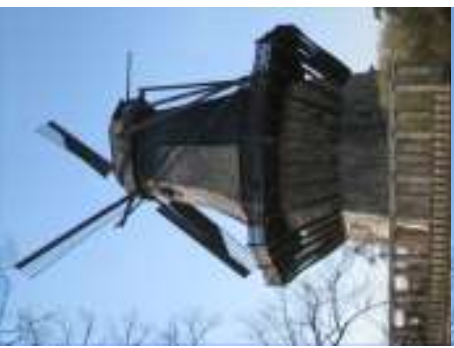

(b)

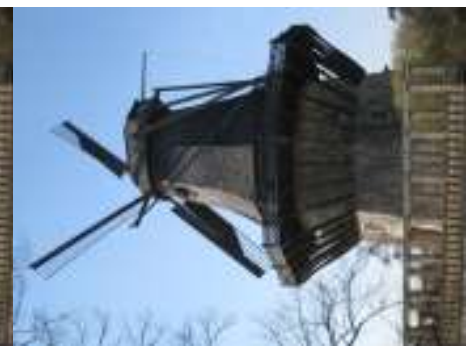

(c)

(d)

(e)

Figure 8. Simulation Results: (a) Original LC \#52 Image, (b) Bilinear Interpolation, (c) Proposed Method, (d) Difference Image Between (a) And (b), and (e) Difference Image Between (a) and (c) 


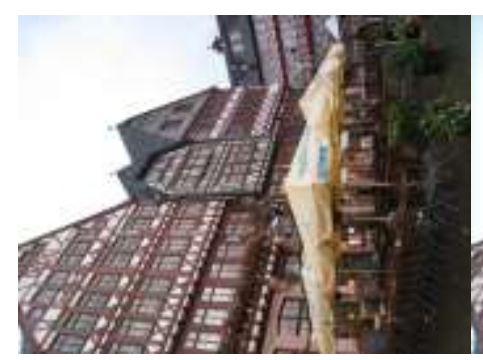

(a)

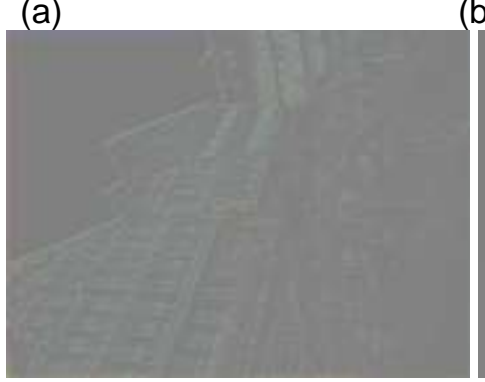

(d)

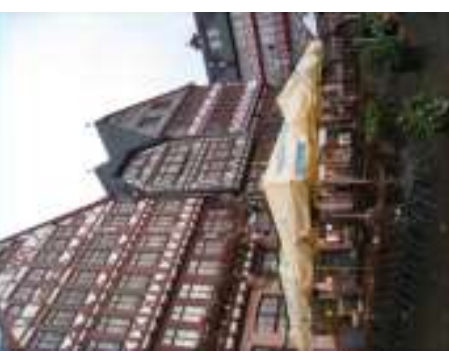

(b)

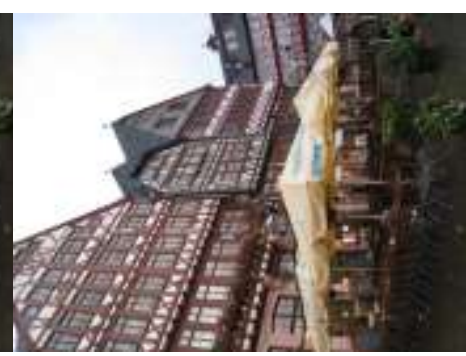

(c)

(e)

Figure 9. Simulation Results: (a) Original LC \#53 Image, (b) Bilinear Interpolation, (c) Proposed Method, (d) Difference Image Between (a) and (b), and (e) Difference Image Between (a) and (c)

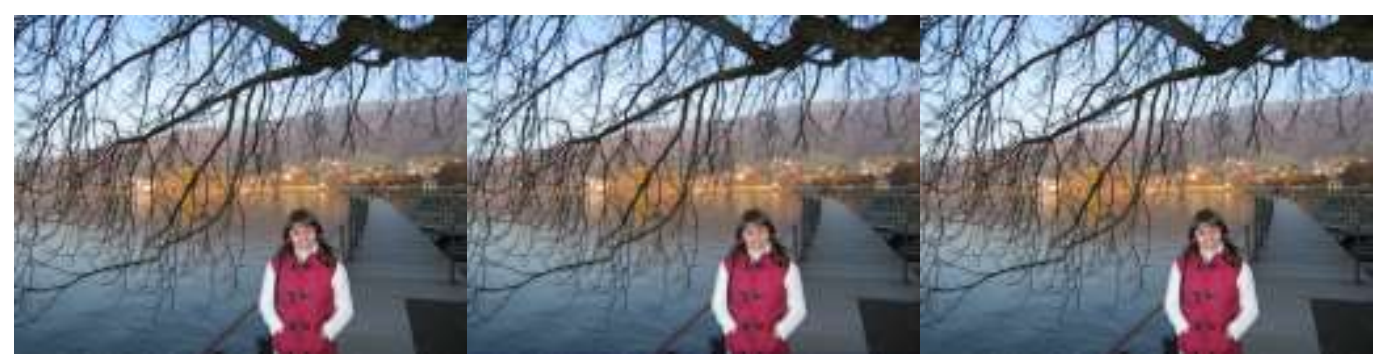

(a)

(b)

(c)

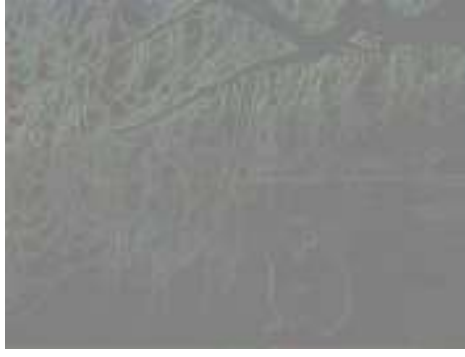

(d) (e)

Figure 10. Simulation Results: (a) Original LC \#54 Image, (b) Bilinear Interpolation, (c) Proposed Method, (d) Difference Image Between (a) and (b), and (e) Difference Image Between (a) and (c) 


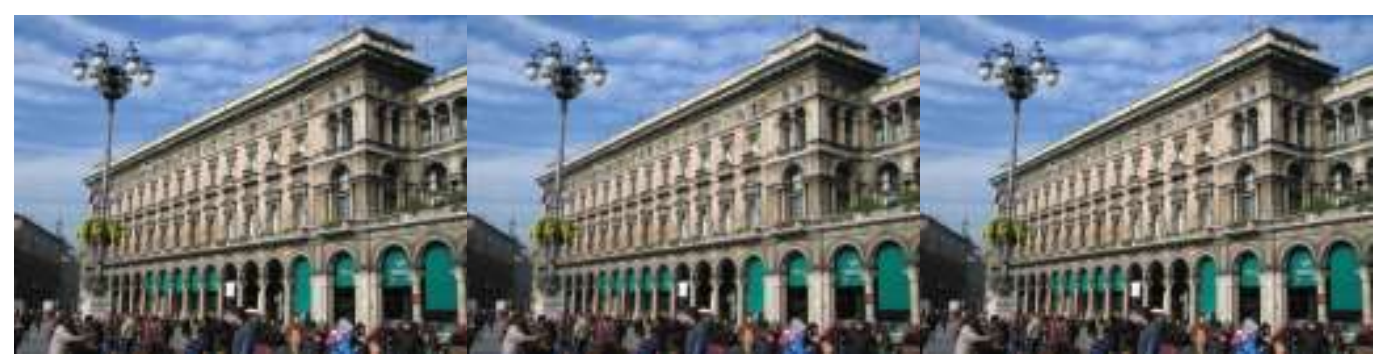

(a)

(b)

(c)

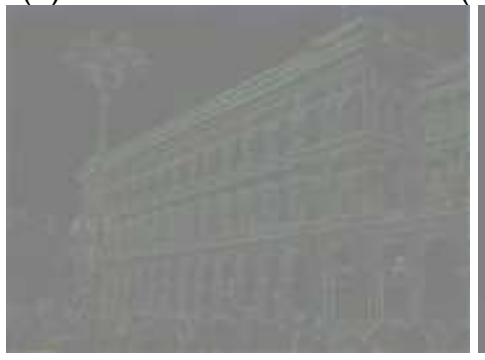

(d)

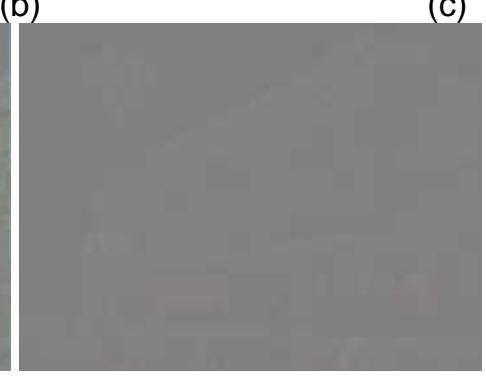

(e)

Figure 11. Simulation Results: (a) Original LC \#55 Image, (b) Bilinear Interpolation, (c) Proposed Method, (d) Difference Image Between (a) and (b), and (e) Difference Image Between (a) and (c)

Table 1 and Table 2 show objective performance comparison in three metrics: CMSE, CPSNR, and S-CIELAB. Table 1 indicates bilinear interpolation results while Table 2 indicates the proposed method results. The same results are displayed in figure form as shown in Figures 12-14, where Figure 12 shows MSE metric, Figure 13 shows PSNR metric, and Figure 14 shows S-CIELAB metric. It is noted that lower MSE, higher PSNR, and lower S-CIELAB indicate better images. It is noted that metrics A to I are as follows:
A: Red channel MSE
F: Green channel PSNR
B: Green channel MSE
G: Blue channel PSNR
C: Blue channel MSE
H: CPSNR
D: CMSE
I: S-CIELAB
E: Red channel PSNR 
Table 1. Objective Performance Results for the Bilinear Interpolation

\begin{tabular}{|c|c|c|r|r|r|r|r|c|c|}
\hline $\begin{array}{c}\text { Image } \\
\text { number }\end{array}$ & $\mathbf{A}$ & $\mathbf{B}$ & $\mathbf{C}$ & $\mathbf{D}$ & $\mathbf{E}$ & $\mathbf{F}$ & $\mathbf{G}$ & $\mathbf{H}$ & $\mathbf{I}$ \\
\hline 51 & 99.65 & 154.66 & 116.69 & 123.67 & 28.14 & 26.23 & 27.46 & 27.20 & 4.11 \\
\hline 52 & 161.16 & 208.53 & 146.30 & 172.00 & 26.05 & 24.93 & 26.47 & 25.77 & 3.19 \\
\hline 53 & 149.12 & 251.27 & 155.56 & 185.32 & 26.39 & 24.12 & 26.21 & 25.45 & 4.74 \\
\hline 54 & 441.81 & 596.80 & 377.00 & 471.87 & 21.67 & 20.37 & 22.36 & 21.39 & 6.42 \\
\hline 55 & 223.34 & 313.57 & 181.78 & 239.57 & 24.64 & 23.16 & 25.53 & 24.33 & 5.41 \\
\hline 56 & 142.66 & 174.09 & 120.21 & 145.65 & 26.58 & 25.72 & 27.33 & 26.49 & 3.66 \\
\hline 57 & 236.04 & 267.63 & 194.28 & 232.65 & 24.40 & 23.85 & 25.24 & 24.46 & 5.18 \\
\hline 58 & 115.31 & 130.56 & 79.37 & 108.41 & 27.51 & 26.97 & 29.13 & 27.77 & 2.75 \\
\hline 59 & 273.51 & 285.55 & 217.53 & 258.86 & 23.76 & 23.57 & 24.75 & 24.00 & 5.00 \\
\hline 60 & 196.44 & 308.53 & 265.57 & 256.85 & 25.19 & 23.23 & 23.88 & 24.03 & 5.53 \\
\hline 61 & 156.99 & 213.14 & 163.44 & 177.86 & 26.17 & 24.84 & 25.99 & 25.63 & 3.24 \\
\hline 62 & 314.88 & 416.68 & 278.36 & 336.64 & 23.14 & 21.93 & 23.68 & 22.85 & 6.50 \\
\hline 63 & 594.22 & 687.65 & 631.83 & 637.90 & 20.39 & 19.75 & 20.12 & 20.08 & 9.11 \\
\hline 64 & 201.28 & 227.50 & 161.99 & 196.92 & 25.09 & 24.56 & 26.03 & 25.18 & 4.29 \\
\hline 65 & 195.74 & 272.66 & 167.61 & 212.00 & 25.21 & 23.77 & 25.88 & 24.86 & 4.76 \\
\hline 66 & 100.66 & 149.56 & 82.79 & 111.00 & 28.10 & 26.38 & 28.95 & 27.67 & 2.80 \\
\hline 67 & 100.97 & 123.83 & 85.26 & 103.35 & 28.08 & 27.20 & 28.82 & 27.98 & 2.74 \\
\hline 68 & 67.07 & 84.22 & 49.96 & 67.08 & 29.86 & 28.87 & 31.14 & 29.86 & 3.11 \\
\hline 69 & 124.79 & 148.55 & 99.22 & 124.19 & 27.16 & 26.41 & 28.16 & 27.18 & 3.42 \\
\hline 70 & 97.76 & 134.48 & 84.10 & 105.45 & 28.22 & 26.84 & 28.88 & 27.90 & 3.56 \\
\hline Avg. & $\mathbf{1 9 9 . 6 7}$ & $\mathbf{2 5 7 . 4 7}$ & $\mathbf{1 8 2 . 9 4}$ & $\mathbf{2 1 3 . 3 6}$ & $\mathbf{2 5 . 7 9}$ & $\mathbf{2 4 . 6 3}$ & $\mathbf{2 6 . 3 0}$ & $\mathbf{2 5 . 5 0}$ & $\mathbf{4 . 4 8}$ \\
\hline & & & & & & & & & \\
\hline
\end{tabular}

Table 2. Objective Performance Results for the Proposed Method

\begin{tabular}{|c|r|r|r|r|r|r|r|c|c|}
\hline $\begin{array}{c}\text { Image } \\
\text { number }\end{array}$ & \multicolumn{1}{|c|}{$\mathbf{A}$} & \multicolumn{1}{|c|}{$\mathbf{B}$} & \multicolumn{1}{c|}{$\mathbf{C}$} & \multicolumn{1}{c|}{$\mathbf{D}$} & $\mathbf{E}$ & $\mathbf{F}$ & $\mathbf{G}$ & $\mathbf{H}$ & $\mathbf{I}$ \\
\hline 51 & 30.85 & 15.26 & 59.72 & 35.28 & 33.23 & 36.29 & 30.36 & 32.65 & 1.54 \\
\hline 52 & 18.55 & 8.56 & 20.00 & 15.70 & 35.44 & 38.80 & 35.11 & 36.16 & 0.90 \\
\hline 53 & 43.18 & 18.63 & 43.64 & 35.15 & 31.77 & 35.42 & 31.73 & 32.67 & 1.45 \\
\hline 54 & 53.63 & 35.53 & 71.99 & 53.72 & 30.83 & 32.62 & 29.55 & 30.82 & 1.89 \\
\hline 55 & 28.23 & 13.38 & 32.81 & 24.81 & 33.62 & 36.86 & 32.96 & 34.18 & 1.61 \\
\hline 56 & 16.31 & 7.92 & 23.24 & 15.83 & 36.00 & 39.13 & 34.46 & 36.13 & 1.06 \\
\hline 57 & 54.24 & 24.68 & 46.76 & 41.90 & 30.78 & 34.20 & 31.43 & 31.90 & 1.99 \\
\hline 58 & 75.75 & 26.59 & 34.51 & 45.61 & 29.33 & 33.88 & 32.75 & 31.53 & 1.80 \\
\hline 59 & 51.62 & 27.61 & 56.93 & 45.39 & 31.00 & 33.72 & 30.57 & 31.56 & 2.26 \\
\hline 60 & 160.60 & 84.72 & 161.00 & 135.44 & 26.07 & 28.85 & 26.06 & 26.81 & 3.25 \\
\hline 61 & 31.59 & 15.45 & 37.22 & 28.09 & 33.13 & 36.24 & 32.42 & 33.64 & 1.17 \\
\hline 62 & 93.38 & 72.34 & 202.52 & 122.75 & 28.42 & 29.53 & 25.06 & 27.24 & 3.96 \\
\hline 63 & 141.77 & 98.43 & 255.52 & 165.24 & 26.61 & 28.19 & 24.05 & 25.94 & 4.88 \\
\hline 64 & 33.95 & 14.04 & 41.15 & 29.71 & 32.82 & 36.65 & 31.98 & 33.40 & 1.46 \\
\hline 65 & 22.86 & 15.36 & 45.14 & 27.79 & 34.53 & 36.26 & 31.58 & 33.69 & 1.75 \\
\hline 66 & 13.44 & 5.95 & 15.34 & 11.58 & 36.84 & 40.38 & 36.27 & 37.49 & 0.82 \\
\hline 67 & 10.35 & 4.64 & 10.55 & 8.51 & 37.98 & 41.46 & 37.89 & 38.82 & 0.72 \\
\hline 68 & 9.65 & 3.05 & 12.92 & 8.54 & 38.28 & 43.27 & 37.01 & 38.81 & 0.79 \\
\hline
\end{tabular}




\begin{tabular}{|c|r|r|r|r|r|r|r|r|r|}
\hline 69 & 18.97 & 6.95 & 19.34 & 15.09 & 35.34 & 39.70 & 35.26 & 36.34 & 1.04 \\
\hline 70 & 34.57 & 15.46 & 33.68 & 27.90 & 32.74 & 36.23 & 32.85 & 33.67 & 1.45 \\
\hline Avg. & $\mathbf{4 7 . 1 7}$ & $\mathbf{2 5 . 7 3}$ & $\mathbf{6 1 . 2 0}$ & $\mathbf{4 4 . 7 0}$ & $\mathbf{3 2 . 7 4}$ & $\mathbf{3 5 . 8 8}$ & $\mathbf{3 1 . 9 7}$ & $\mathbf{3 3 . 1 7}$ & $\mathbf{1 . 7 9}$ \\
\hline
\end{tabular}

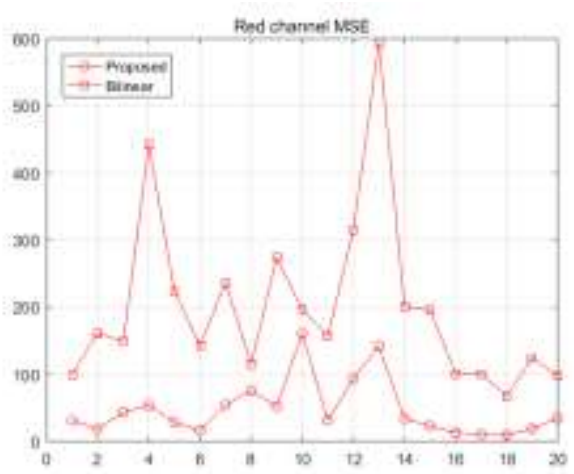

(a)

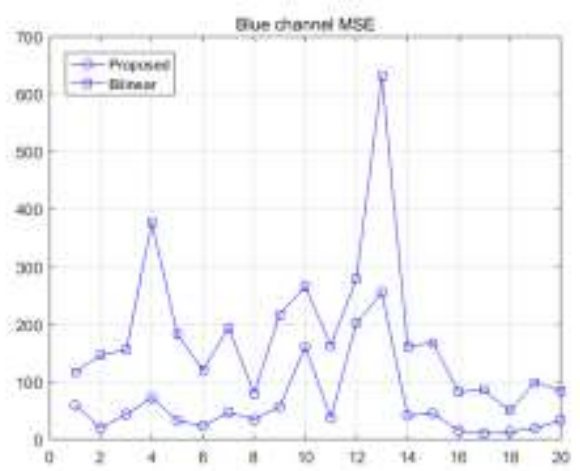

(c)

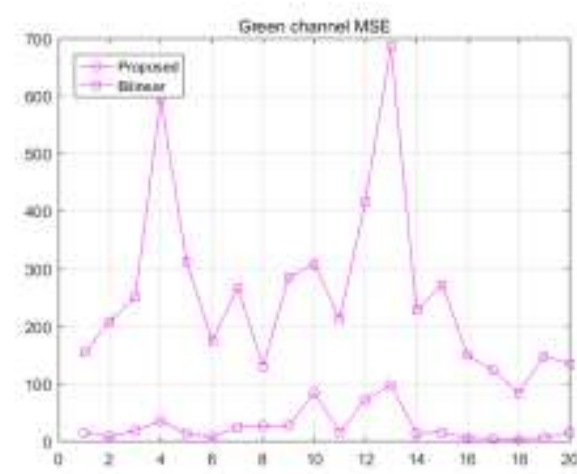

(b)

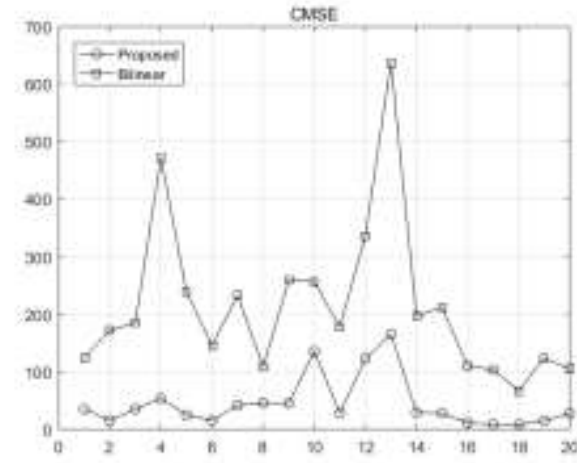

(d)

Figure 12. Objective Performance Comparison in Terms of MSE: (a) Red Channel, (b) Green Channel, (c) Blue Channel, and (d) CMSE

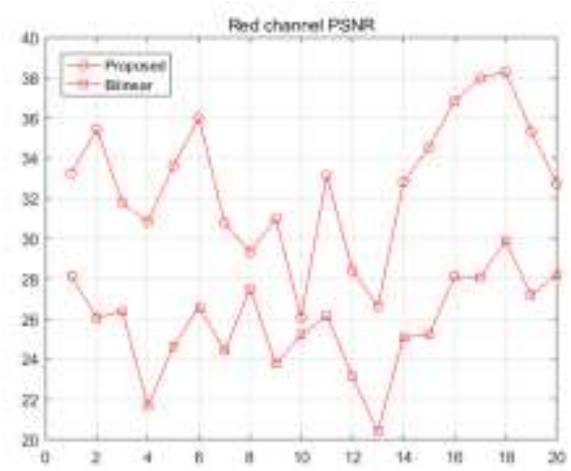

(a)

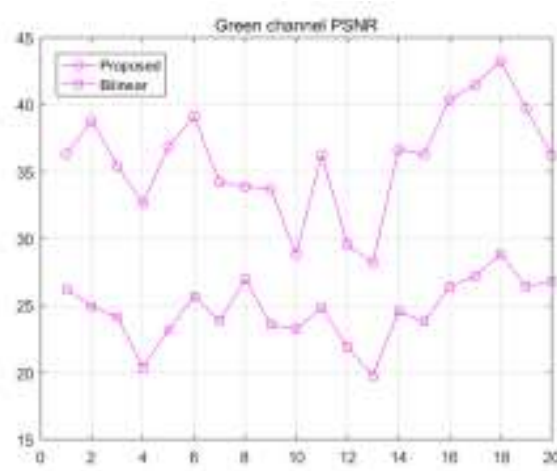

(b) 


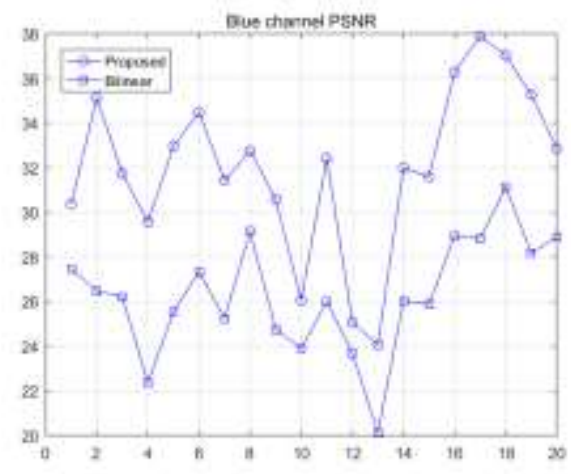

(c)

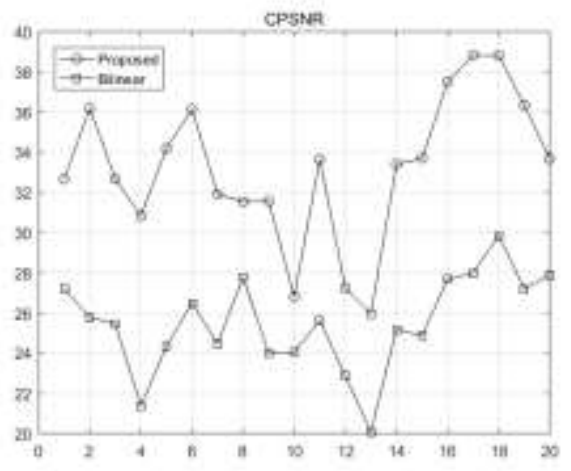

(d)

Figure 13. Objective Performance Comparison in Terms of PSNR: (a) Red Channel, (b) Green Channel, (c) Blue Channel, and (d) CPSNR

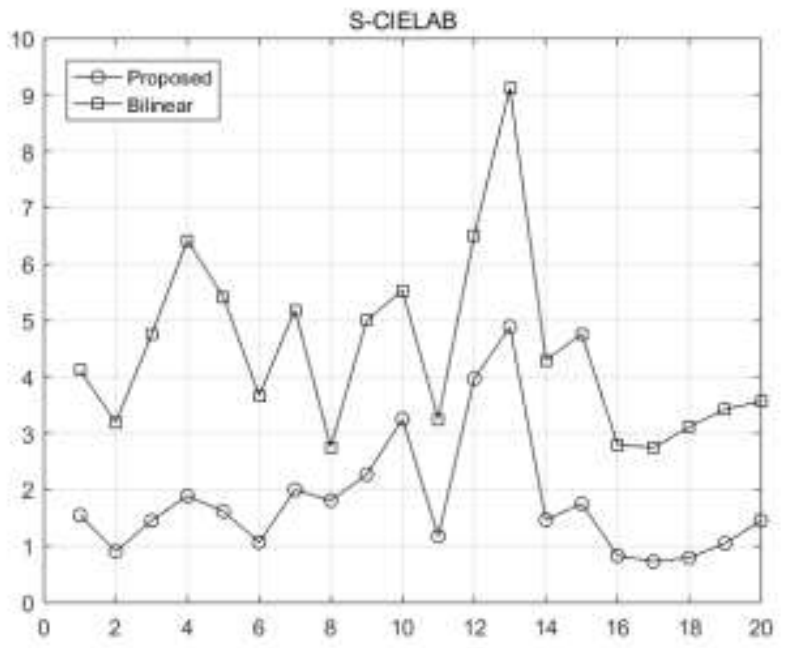

Figure 14. Objective Performance Comparison in Terms of S-CIELAB

\section{Conclusions}

Due to the cost reason, current digital cameras adopt color filter array instead of beam splitters of full-color RGB camera. Although RGB camera provides more natural images, but its beam splitters for each pixel may cause high costs. Therefore, usage of CFA is inevitable and its color interpolation (demosaicking) is important topic. The proposed method restores color image from a given CFA image.

The proposed method has three steps: (1) green channel interpolation, (2) red/blue channel interpolation, and (3) all channels refinement. As green component has more information than that of red or blue components, it is suggested to restore green channel first before red or blue channel. Simulation results inform that lower MSE, higher PSNR, and lower S-CIELAB indicate better images. Therefore, the proposed approach gives reliable results in terms of visual and objective performance.

\section{Acknowledgments}

This research was supported by 2016-2 Hanyang Women's University Research Fund. This paper is a revised and expanded version of a paper entitled "Subsampled Channel Difference for Color Image Enhancement" presented at GST 2016. 


\section{References}

[1] D. Menon and G. Calvagno, "Color image demosaicking: an overview", Signal Processing: Image Communication, vol. 26, no. 8-9, (2011), pp. 518-533.

[2] X. Li, B. K. Gunturk, L. Zhang, "Image demosaicing: a systematic survey", Proc. SPIE 6822, Visual Communications and Image Processing, San Jose, CA, (2008), pp. 68221J1-68221J15.

[3] D. R. Cok, "Signal processing method and apparatus for producing interpolated chrominance values in a sampled color image signal", U.S. Patent 4,642,678, (1987) February 12.

[4] J. E. Adams, "Intersections between color plane interpolation and other image processing functions in electronic photography”, Proc. SPIE, vol. 2416, (1995), pp. 144-151.

[5] J. Hamilton and J. Adams, "Adaptive Color Plane Interpolation in Single Sensor Color Electronic Camera", U.S. Patent 5,629,734, (1997) May 13.

[6] D. Menon, S. Andriani, and G. Calvagno, "Demosaicing with Directional Filtering and a Posteriori Decision”, IEEE Transactions on Image Processing, vol. 16, no. 1, (2007), pp. 132-141.

[7] Z. Dengwen, S. Wxialiu, and Weiming, "Colour Demosaicking with Directional Filtering and Weighting”, IET Image Processing, vol. 6, no. 8, (2012), pp. 1084-1092.

[8] W.-J. Chen and P.-Y. Chang, "Effective Demosaicking Algorithm based on Edge Property for Color Filter Arrays", Digital Signal Processing, vol. 22, no. 1, (2012), pp. 163-169.

[9] L. Wang and G. Jeon, "Bayer Pattern CFA Demosaicking based on Multi-directional Weighted Interpolation and Guided Filter," IEEE Transactions on Signal Processing Letters, vol. 22, no. 11, (2015), pp. 2083-2087.

[10] J. Kim, G. Jeon, and J. Jeong, "Demosaicking using Geometric Duality and Dilated Directional Differentiation", Optics Communications, vol. 324, (2014), pp. 194-201.

[11] S. Kim, "An Error Concealment Technique Based on Directional Interpolation”, International Journal of Applied Engineering Research, vol. 10, no. 13, (2015), pp 33677-33679.

[12] D. Kim, "Weighted Histogram Equalization Method adopting Weber-Fechner's Law for Image Enhancement", Journal of the Korea Academia-Industrial cooperation Society, vol. 15, no. 7, (2014), pp. 4475-4481.

[13] S. Kim, "Medical Image Enhancement Using an Adaptive Nonlinear Histogram Stretching", Journal of the Korea Academia-Industrial cooperation Society, vol. 16, no. 1, (2015), pp. 658-665.

[14] J. Lee, S. Jeong, and J. Lee, "Photo Mosaic Generation Algorithm Using the DCT Hash", Journal of the Korea Academia-Industrial cooperation Society, vol. 17, no. 10, (2016), pp. 61-67.

[15] S. Kim, "Adaptive Camera Image Interpolation Method using Directional Color Plane Information", SERSC International Journal of Multimedia and Ubiquitous Engineering, vol. 11, no. 6, (2016), pp.143152.

[16] S. Kim, "Subsampled Channel Difference for Color Image Enhancement", Proceedings of the $3^{\text {rd }}$ International Mega-Conference on Green and Smart Technology, Jeju, Korea, (2016) December 21-23.

[17] X. Wu and N. Zhang, "Primary-consistent Soft-decision Color Demosaicking for Digital Cameras", IEEE Transactions on Image Processing, vol. 13, no. 9, (2004), pp. 1263-1274.

[18] J. Wang, J. Wu, Z. Wu, and G. Jeon, "Taylor Series and Adaptive Directional Selection for Real Time Demosaicking,” Displays, vol. 45, (2016), pp. 14-25.

[19] G. Jeon, "Noise Model Analysis on Image Sensor Arrays", SERSC International Journal of Multimedia and Ubiquitous Engineering, vol. 10, no. 7, (2015), pp.93-104.

[20] G. Jeon, "Design of Multiple Channel Color Filter Array", Journal of Engineering Technology, vol. 3, no. 1, (2015), pp. 34-44. 


\section{Author}

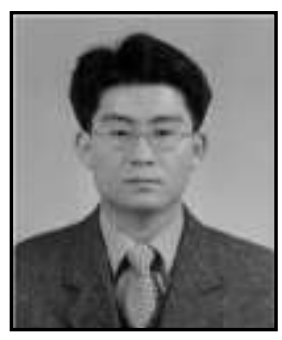

Seungjong Kim, He received the M.S. and Ph.D. degrees in Electronic Communications Engineering from Hanyang University, Seoul, Korea, in 1994 and 2000, respectively. Since 2000, he is a professor of the Department of Computer Science \& Information Systems at Hanyang Women's University, Seoul, Korea. His research interests include digital signal processing, digital communication, and image processing for multimedia applications. He received the ETRI Journal paper award with the "Error Concealment Using Intra-Mode Information Included in H.264/AVC-Coded Bitstream" in January 2009. He received the "49th Annual Trade Day" award from the Prime Minister of Korea in December 2012. 
International Journal of Signal Processing, Image Processing and Pattern Recognition Vol. 10, No. 7 (2017) 